\title{
Imaging vasculature independent of direction of flow using spectral domain optical coherence tomography
}

J. Fingler, J. Williams, C. Yang, S. E. Fraser

J. Fingler, J. Williams, C. Yang, S. E. Fraser, "Imaging vasculature independent of direction of flow using spectral domain optical coherence tomography," Proc. SPIE 6429, Coherence Domain Optical Methods and Optical Coherence Tomography in Biomedicine XI, 64290P (7 February 2007); doi: 10.1117/12.697231

SPIE. Event: SPIE BiOS, 2007, San Jose, California, United States 


\title{
Imaging Vasculature Independent of Direction of Flow using Spectral Domain Optical Coherence Tomography
}

\author{
J. Fingler, J. Williams, C. Yang, S. E. Fraser \\ California Institute of Technology, Pasadena, CA, 91125
}

\begin{abstract}
A variation to the analysis of phase data achieved with spectral domain optical coherence tomography (SDOCT) is presented. By using the variance of the phase changes observed in the OCT images, scatterer motion has been imaged which is not readily observable with conventional Doppler OCT techniques. Dynamic motion contrast has been demonstrated for imaging Brownian motion of a sample system as well as imaging vasculature of in vivo $3 \mathrm{dpf}$ zebrafish.
\end{abstract}

\section{INTRODUCTION}

In spectral domain optical coherence tomography (SDOCT), the intensity and phase of each depth resolved reflection can be obtained all at the same time in what is defined as an A-scan, limited in speed only by the spectrometer acquisition time $\tau$. The most common form of motion contrast developed with this phase information is the mean axial flow rate (flow along the imaging direction), which is determined from the average phase change of reflections at a given depth $\mathrm{z}$ from A-scans separated by time $\mathrm{T}:{ }^{[1-3]}$

$$
v_{\text {axial }}(z)=\frac{\lambda}{4 \pi n T}\langle\phi(z, t+T)-\phi(z, t)\rangle=\frac{\lambda}{4 \pi n T}\langle\Delta \phi(T)\rangle
$$

In this definition, $\lambda$ is the mean wavelength of the light source and $\mathrm{n}$ is the refractive index of the material where the phase measurement was taken. Many groups perform flow imaging using successive A-scans to measure phase changes such that $T=\tau$, while performing time averaging over a few measurements (typically 4 or 5). The maximum axial flow component that can be determined is limited by phase wrapping, which limits the maximum velocity component measurement to $v_{\text {axial,max }}(z)= \pm \lambda / 4 n \tau$.

This method only determines the flow component along the imaging direction of the system designated by $v(\cos \vartheta)$, where v is the velocity of the flow and $\vartheta$ is the angle of the flow direction relative to the imaging direction. For cases such as in the retina, the majority of the flow is nearly perpendicular to the imaging direction such that $\vartheta \sim 90^{\circ}$. In these cases, the velocity v of the flow must be extremely high to be able to visualize the flow with this method.

Methods demonstrating the use of phase variance in identifying vasculature utilize only a few successive A-scans and have so far demonstrated limited contrast in their current implementation. The techniques demonstrated with the most significant contrast are sacrificing imaging resolution to accomplish this and are not able to visualize very small vessels with their methods. ${ }^{[3,4]}$

\subsection{Phase Changes}

\section{METHODS AND THEORY}

The phase change measured for a given depth $z$ with a time separation $\mathrm{T} \Delta \phi(z, T)$ has a combination of several factors effecting the accuracy of the measurement:

$$
\Delta \phi(z, T)=\Delta \phi_{\text {motion,scatterer }}(z, T)+\Delta \phi_{\text {motion,bulk }}(T)+\Delta \phi_{\text {error }, S N R}(z)+\Delta \phi_{\text {error, other }}(z)
$$


The motion of interest is from the scatterers located at the depth $\mathrm{z}$, whose motion is designated by the phase change term $\Delta \phi_{\text {motion,scatterer }}(z, T)$. In this case, the phase change $\Delta \phi(z, T)$ also contains the bulk motion of the entire sample along the imaging (axial) direction $\Delta \phi_{\text {motion,bulk }}(T)$, caused by relative phase motion between the sample and the system. $\Delta \phi_{\text {error }, S N R}(z)$ designates a phase error associated with the OCT SNR of the data calculated at the depth $z$. Experimental and theoretical results have determined that the phase variance of SNR-limited phase error for phase changes has the form: ${ }^{[3,5,6]}$

$$
\sigma_{\triangle \phi, S N R}(z)=1 / \sqrt{S N R_{O C T}(z)}
$$

$\Delta \phi_{\text {error,other }}(z)$ encompasses the other phase errors which may occur for OCT phase measurements, including but not limited to phase changes caused by transverse motion ${ }^{[3]}$, artifacts associated with limited depth sampling during axial motion of the sample, or other computational based phase anomalies.

By understanding the effects $\Delta \phi_{\text {motion,bulk }}(T), \Delta \phi_{\text {error }, S N R}(z)$, and $\Delta \phi_{\text {error, other }}(z)$ have on the accuracy of phase measurements in an OCT system, many improvements can be made to improve the dynamic range of demonstrated phase contrast techniques and we gain the ability to move beyond these techniques for new contrast opportunities.

\subsection{Bulk Phase Removal}

Without any compensation, phase measurements for a given depth would have a difficult time separating the motion between the motion of a given scatterer and the bulk motion of the system and sample. In cases which contain a high reflection interface, this surface can be used as a reference to remove off all bulk axial motion of the sample and system. For specific cases where a reference reflector is not available or not ideal for phase removal of bulk motion, the phase changes at all of the sample depths can be used.

We used a weighted mean technique to calculate the bulk motion, involving individual phase changes from different depths. Each phase change calculated was weighted by the intensity of the OCT signal for that depth to reduce the effect SNR-limited phase error $\sigma_{\Delta \phi, S N R}(z)$ has on the calculation of the bulk motion removal. For phase measurements separated by time T, the bulk axial motion is calculated:

$$
\Delta \phi_{\text {motion,bulk }}(T)=\sum_{z}[I(z) \Delta \phi(z, T)] / \sum_{z}[I(z)]
$$

The intensity $I(z)$ used in the bulk motion calculation is the linear value of the imaged OCT intensity, which is defined by $20 \log (I(z))$ in this system. The summation for this equation is over a chosen depth region of the image which contains the sample. The phase changes $\Delta \phi(z, T)$ used were conditioned to limit phase changes between $-\pi$ and $+\pi$ to avoid phase wrapping issues. With this method of bulk phase removal from the entire depth sample reflections, the accuracy of this method becomes limited to approximately the SNR of the strongest sample reflection in the entire depth. This places an expected lower limit on measurable phase changes after compensating for the other effects.

Figure 1 demonstrates the effect of using phase changes from multiple depths within a paper sample for a given bulk phase change with no weighting based on intensity. The histograms in (a) and (b) show the distribution of calculated phase changes for a bulk motion of approximately 1.5 radians, applying (a) no threshold on the OCT data and using (b) a threshold to limit data to $15 \mathrm{~dB}$ over the OCT mean noise level. Figure 1 (c) demonstrates the effect the threshold amplitude has on the standard deviation of the phase changes. 

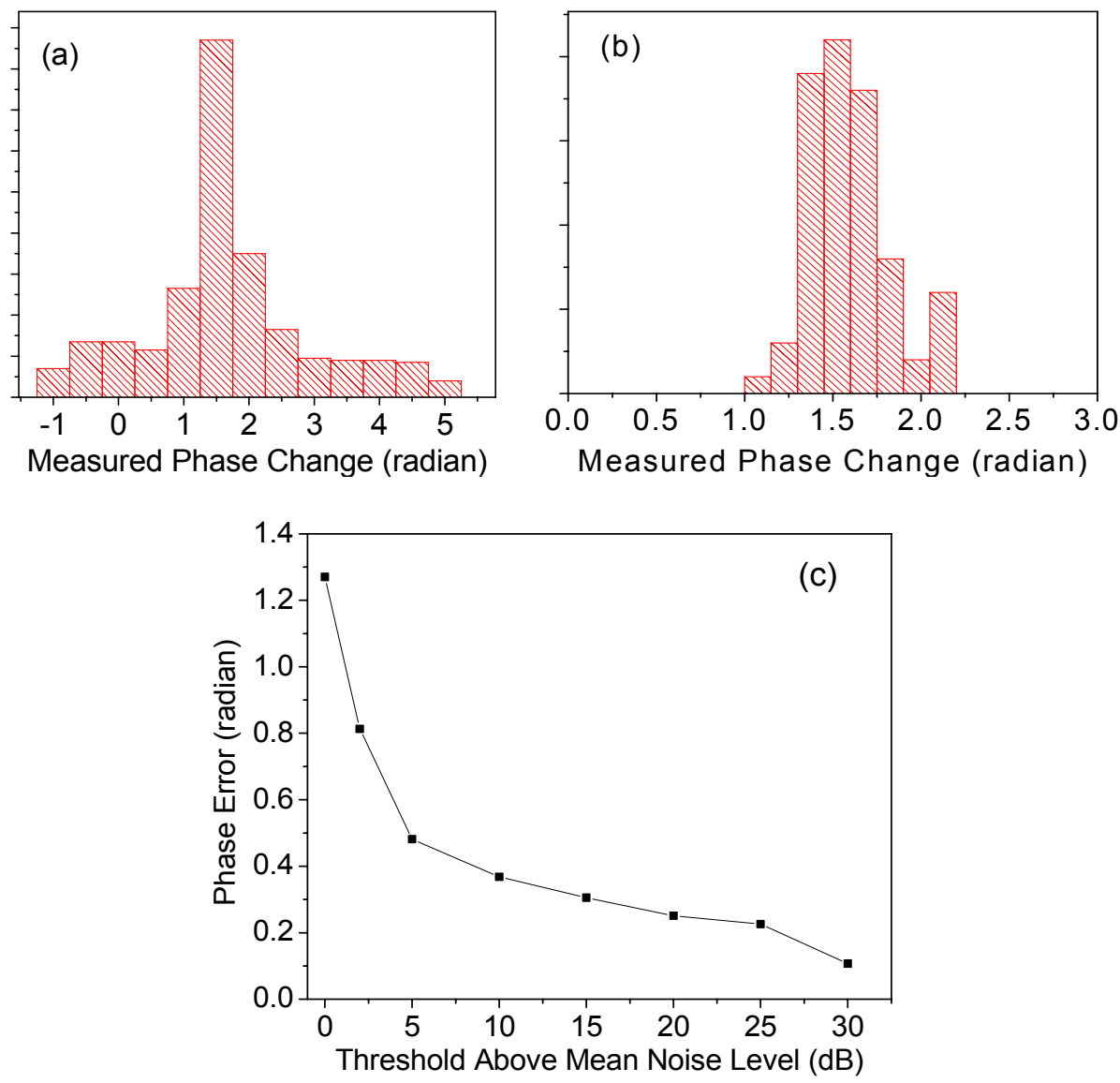

Figure 1. Calculated phase changes over OCT A-scans of paper sample for approximately 1.5 radians of bulk motion. The histograms in (a) and (b) present a distribution of phase changes calculated for a region of the A-scan, for cases with no threshold applied and a threshold applied for OCT signal $15 \mathrm{~dB}$ higher than the calculated noise level, respectively. The standard deviation calculated for the data in (a) and (b) and 1.27 radians and 0.31 radians, respectively. Graph (c) plots the calculated standard deviation of the phase change measurements for different OCT intensity thresholds applied.

\subsection{Phase Variance Contrast}

With the compensation of the bulk sample motion, and assuming no correlation between different phase noise terms, the phase variance calculated from the corrected phase change $\Delta \phi(z, T)-\Delta \phi_{\text {motion,bulk }}(T)$ appears of the form:

$$
{\sigma_{\Delta \phi}}^{2}(z, T)=\sigma_{\Delta \phi, \text { scatterer }}^{2}(z, T)+{\sigma_{\Delta \phi, S N R}}^{2}(z)+{\sigma_{\text {error_bulk }}}^{2}(z)+\sigma_{\text {error,other }}{ }^{2}(z)
$$

The primary contrast information is $\sigma_{\Delta \phi \text {,scatterer }}{ }^{2}(z, T)$, so it is important to be able to reduce or remove the effects of the other phase noise terms to improve contrast imaging. Since there is no time dependence on the main phase noise terms, increasing the time between phase measurements allows for an increase in the motion signal $\sigma_{\Delta \phi, \text { scatterer }}{ }^{2}(z, T)$ while keeping the noise constant. 
For 2 phase measurements separated by time $T$ :

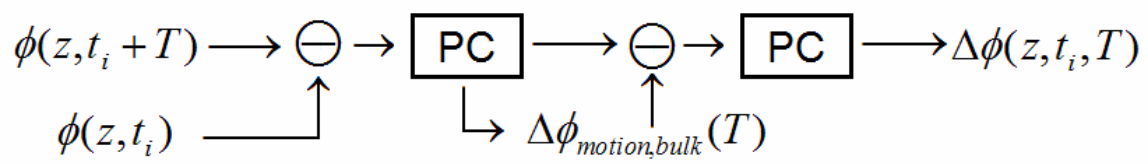

Repeated for $N$ phase changes:

$$
\left\{\Delta \phi\left(z, t_{i}, T\right)\right\}_{i=1, \ldots, N}^{\longrightarrow} \sigma_{\Delta \phi}^{2}(z, T)
$$

Figure 2. Schematic of phase analysis technique used to produce phase variance contrast.

PC: phase conditioning to limit maximum phase change between measurements.

Two different scan modalities are presented to allow acquisition of 2D phase contrast images with similar time regimes, which are referred to as MB scans and BM scans in this paper. Referencing OCT scanning terminology, an A-scan refers to a single reflectivity depth scan in the sample and a B-scan refers to multiple A-scans taken over different transverse positions to create a 2D OCT image. An M-scan refers to multiple A-scans taken over time at the same transverse location. For the purpose of this paper, an MB-scan is a term to describe multiple M-scans taken over transverse positions to create a 2D OCT image of Mscans. A BM-scan on the other hand is multiple B-scans taken over time for the same scan region. A graphical representation of a portion of a sample scan pattern for a MB-scan and a BM-scan is shown in Figure 3.
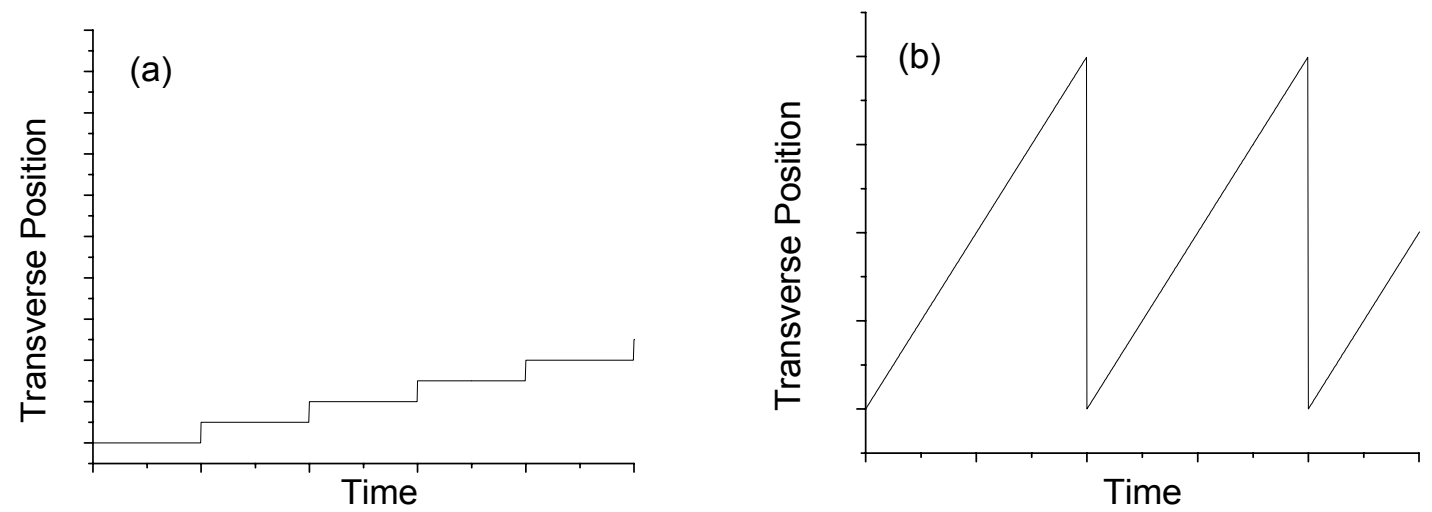

Figure 3. Schematic of transverse scan patterns for the (a) MB scan and (b) BM scan

\subsection{MB Scan}

The purpose of the MB scan is to acquire additional temporal information of the scatterer motion to improve motion contrast. Increasing the amount of A-scans taken within each M-scan not only increases the maximum time between phase images that can be measured, but the improved statistics can increase the dynamic range of standard Doppler flow imaging techniques as mentioned in equation (1).

The basic contrast metric used for MB scans with this system is $\sigma_{\Delta \phi}{ }^{2}\left(z, T_{2}\right)-\sigma_{\Delta \phi}{ }^{2}\left(z, T_{1}\right)$, such that $T_{2} \gg T_{1}$ and the calculated value is the scatter motion $\sigma_{\Delta \phi \text {,scatterer }}{ }^{2}\left(z, T_{2}\right)-\sigma_{\Delta \phi, \text { scatterer }}{ }^{2}\left(z, T_{1}\right)$. For the example of Brownian motion of diffusing spheres, the expected form of the variance of the scatterer motion is $\sigma_{\Delta \phi, s c a t t e r e r}{ }^{2}(z, T)=D T$. The phase contrast $\sigma_{\Delta \phi}{ }^{2}\left(z, T_{2}\right)-\sigma_{\Delta \phi}{ }^{2}\left(z, T_{1}\right)$ removes the SNR limiting error and leaves a phase contrast of $D\left(T_{2}-T_{1}\right) \approx D T_{2}$ for $T_{2} \gg T_{1}$. 


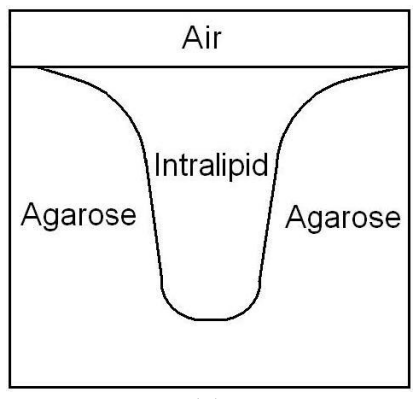

(a)

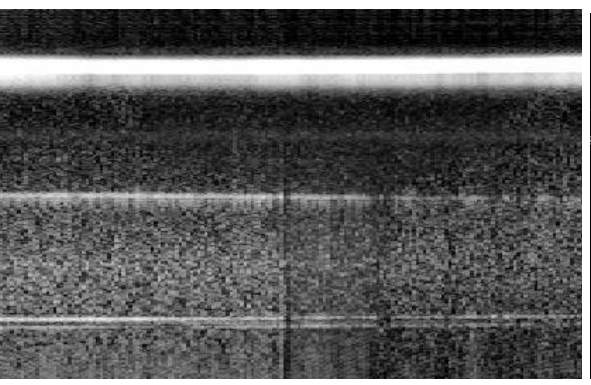

(b)

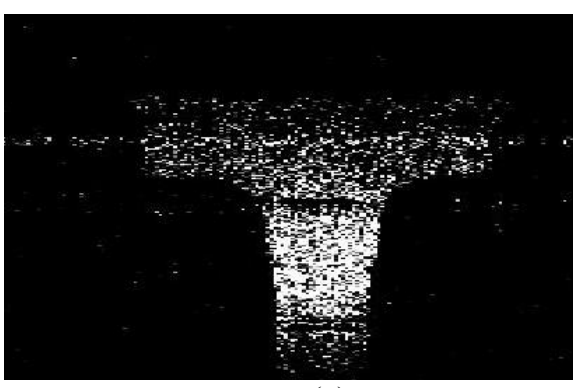

(c)

Figure 4. Cross-sectional images of $2 \%$ agarose wells filled with $0.04 \%$ Intralipid. The schematic image in (a) describes the expected form of the filled wells. The OCT intensity image (b) shows limited contrast in visualizing the well due to scattering intensity matching. Artifacts which appear in this image are due to multiple reflections and CCD camera noise. The phase contrast image (c) uses a threshold to eliminate high phase noise terms from the image.

Figure 4 demonstrates the visualization of scatterers undergoing motion similar to Brownian motion. Figure 4(a) shows a schematic of a $2 \%$ agarose well which has been filled with $0.04 \%$ Intralipid, chosen in concentration to match the agarose scattering signal intensity. Figure 4(b) shows an OCT intensity image of the filled agarose well as described in the schematic, demonstrating the limited intensity contrast. The phase contrast $\sigma_{\Delta \phi}{ }^{2}\left(z, T_{2}\right)-\sigma_{\Delta \phi}{ }^{2}\left(z, T_{1}\right)$ is used in figure 4(c) with $T_{2}=1 m s$ and $T_{1}=40 \mu s$. The threshold of $\sigma_{\Delta \phi}{ }^{2}(z, 40 \mu s) \geq 1$ applied to the contrast image corresponds to $\operatorname{SNR}_{O C T}(z) \leq 1$ as described by equation (3). Artifacts which appear in the OCT image cause false phase contrast (negative and positive) due to motion compensation on artifacts which do not change in the same way as the bulk sample or the individual scatterers.

The MB scan is an extension of conventional OCT Doppler imaging techniques which can require significantly more time per transverse position compared to the original technique in order to get adequate motion contrast. This limits the ultimate speed for imaging with this technique because increased acquisition speed through technological advances will still require the same imaging time to get the same motion contrast. Observation of flow pulsatility is limited by phase contrast image speed, which for demonstrated contrast images in this manuscript is 200 times slower than a conventional OCT image of the same size.

\subsection{BM Scan}

The expected motion contrast associated with flow and Brownian-type motion increases with an increased time separation between phase measurements. By using the phase differences between successive B-scans, the data throughput of phase contrast images is increased and the possible image contrast is increased as well. In order to ensure a constant time separation $\mathrm{T}$ between phase measurements for each transverse location and maintain consistency of contrast across the image, successive B-scans are used for transverse scans of the same direction (every $2^{\text {nd }}$ image from bi-directional scanning).

The phase contrast metric $\sigma_{\Delta \phi}{ }^{2}\left(z, T_{2}\right)-\sigma_{\Delta \phi}{ }^{2}\left(z, T_{1}\right)$ used for MB scans is not ideal for this situation due to the fact that the phase error between successive A-scans $\sigma_{\Delta \phi}{ }^{2}\left(z, T_{1}\right)$ for $T_{1}=40 \mu$ s contains additional phase errors $\sigma_{\text {error, other }}{ }^{2}(z)$ mentioned in equation (5). These additional errors occur due to the transverse 
scanning occurring during successive A-scans to create each B-scan. ${ }^{[3]}$ One of the ways to compensate for $\sigma_{\triangle \phi, S N R}^{2}(z)$ is to calibrate an expected estimate of the phase error based on theory.

The spectrometer measures the contributions from interferometric signal from the OCT signal as well as the noise sources in the system. With signal from two independent sources, the complex FFT of the spectrometer signal calculates a complex value based on the combination of these sources:

$$
\widetilde{I}=I e^{i \phi}=S e^{i \phi_{S}}+N e^{i \phi_{N}}
$$

The intensity amplitude $I$ is the same as used in equation (4) for bulk motion calculation, so the OCT intensity is of the form $20 \log (I)=10 \log \left(|\widetilde{I}|^{2}\right)$. The sample signal is designated with an amplitude $\mathrm{S}$ and a relative phase to the reference arm of $\phi_{S}$. For this theory, it is assumed at the noise amplitude $\mathrm{N}$ remains constant and the phase of the noise term $\phi_{N}$ is completely random. The magnitude of the intensity is calculated as:

$$
|\widetilde{I}|^{2}=I^{2}=S^{2}+N^{2}-2 S N \cos \left(\phi_{S}-\phi_{N}\right)
$$

Since the phase of the noise term is completely random, time averaging the magnitude of the intensity leaves us with $\left\langle|\widetilde{I}|^{2}\right\rangle=S^{2}+N^{2}$. Combining this formalization with the definition of SNR-limited phase error from equation (3):

$$
\sigma_{\triangle \phi, S N R}^{2}(z)=1 / S N R_{O C T}(z)=N^{2} / S(z)^{2}=1 /\left[\left(S(z)^{2}+N^{2}\right) / N^{2}-1\right]=1 /\left(\left[\left\langle\left.\tilde{I}(z)\right|^{2}\right\rangle / N^{2}\right]-1\right)
$$

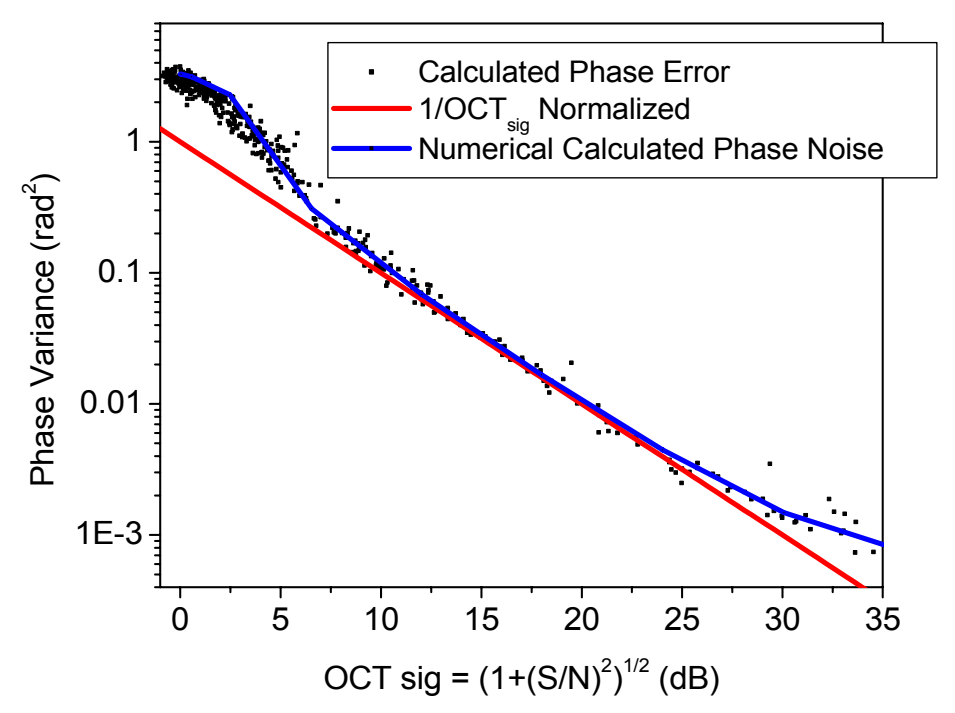

Figure 5. Phase Error calculated as a function of OCT signal. Linear fit (straight line) was performed to data in $10 \mathrm{~dB}$ to $25 \mathrm{~dB}$ range to estimate noise value in system. Numerical estimates of phase error (curved line) are based on noise value calculations, approximate bulk motion error values and limiting possible phase changes to within $-\pi$ to $\pi$ radians. 
Estimation of the noise level $N^{2}$ of the OCT system was achieved by fitting the OCT signal for the data in the linear regime where $\mathrm{S}>>\mathrm{N}$ and is negligibly affected by the bulk motion compensation phase error, which in this case was $10-25 \mathrm{~dB}$ above the noise level. Using this value and equation (9), the SNRlimiting phase error is estimated. Due to the imposed limitations of maximum phase changes between $-\pi$ and $+\pi$, there is a maximum expected phase error to be calculated. For a completely random distribution of phase changes between $-\pi$ and $+\pi$, the standard deviation is approximately 1.8 radians. This limits the expected maximum phase variance measured for a purely noise situation to approximately 3.2 radians $^{2}$. For cases where $\mathrm{S} / \mathrm{N}<1$, the limitations on maximum phase changes causes equation (9) to overestimate the expected phase error. For these very low $\mathrm{S} / \mathrm{N}$ cases, numerical estimates are required which, like our analysis, limit that maximum phase change between successive measurements to be between $-\pi$ and $+\pi$. Figure 5 plots the calculated phase error from 200 successive A-scans as a function of the average OCT signal, along with the linear fit used to determine the noise value of the system and the numerical estimate of the phase error based on maximum limitations to phase changes.

Since in BM scans the time between B-scans $\mathrm{T}$ is much larger than the spectrometer integration time $\tau$, the maximum velocity that can be properly measured using traditional Doppler OCT methods is severely limited due to phase wrapping. Using the phase variance contrast of the scatterers, three main types of motion may still be observed: (i) the axial component of motion caused by Brownian-type random motion, (ii) phase effects due to transverse motion of the sample ${ }^{[3]}$, and (iii) variations in the axial flow over a time period $\mathrm{T}$. The combination of all of these effects on the phase variance measurements will help to visualize locations of blood flow independent of the direction of flow relative to the imaging system.

\subsection{Zebrafish Vasculature Imaging}

For the first in vivo studies of vasculature imaging, 3-4 dpf (days post-fertilization) zebrafish were used. Zebrafish (Danio rerio) is ideal for imaging due to the fully developed vasculature system and the optical transparency of the animal. Figure 6 demonstrates the anatomy and vasculature of a typical $3 \mathrm{dpf}$ zebrafish through confocal images and histological sections. Figure 6 (b) is an image produced of a zebrafish strain designed to express GFP (green fluorescent protein) in its vasculature for improved visualization. The lines appearing on figures 6 (a) and (b) depict the anatomical location where the histological section of (c) was taken from. The locations of the primary blood vessels of the zebrafish tail (dorsal aorta and axial vein) are labeled along with the major anatomical features on the image.

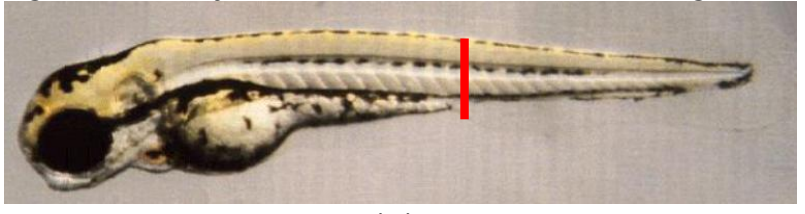

(a)

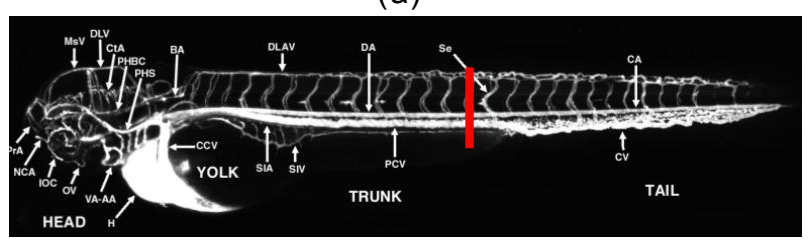

(b)

Figure 6. Images of 3 dpf zebrafish ${ }^{[7,8]}$ (a) Brightfield con fo cal image. (b) Con fo cal image of GFP-lab elled vasculature. (c) Histo logy slice of zebrafish correspond ing to lo cation depicted in (a) and (b). Dorsal side of fish orien ted to wards the top of each images

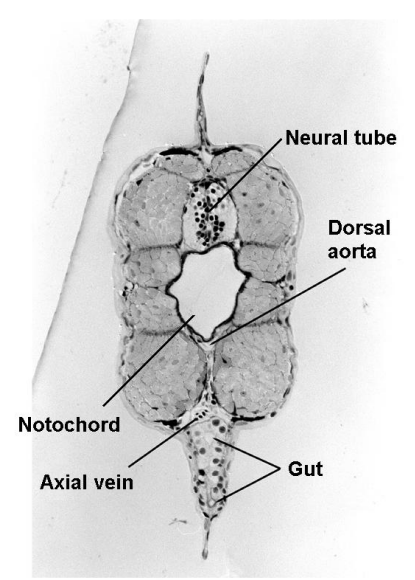

(c) 
In this experiment, zebrafish embryos earlier than $1 \mathrm{dpf}$ had phenylthiourea (PTU) applied to them, a chemical used routinely to inhibit pigment production in zebrafish. After allowing the zebrafish to mature to the 3-4 dpf stage, the zebrafish to be imaged were anesthetized using a Tricaine solution and positioned within agarose wells filled with egg water. The most natural position of the zebrafish within these wells was to lay on its side, allowing for confocal images similar to shown in figure 6 . This alignment allowed for easier accessibility to transversely separate blood vessels, especially in the tail. For the images shown in this manuscript, a cover slip was used on the egg water-filled agarose wells containing the zebrafish.

For the spectral domain OCT system used to image these zebrafish, the maximum measured resolution was $6 \mu \mathrm{m}$ transversely with a $7 \mu \mathrm{m}$ coherence length in air, which corresponds to approximately $5 \mu \mathrm{m}$ in tissue. The spectrometer acquisition time limited the minimum time between A-scans to $40 \mu \mathrm{s}$. Figure 7 presents images analyzed from MB-scan data taken from 4dpf zebrafish imaged across the tail at an approximate location depicted by the scan lines in figure 6 (a) \& (b). The phase contrast image in figure 7 (b) uses the data analysis technique of the phase variance described in section 2.4. The motion contrast images (b)-(d) uses the same threshold on data as used in figure 4 . All images shown were dimensionally scaled and cropped to highlight the region of interest.

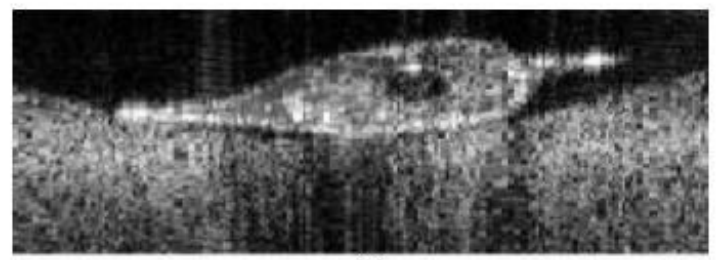

(a)

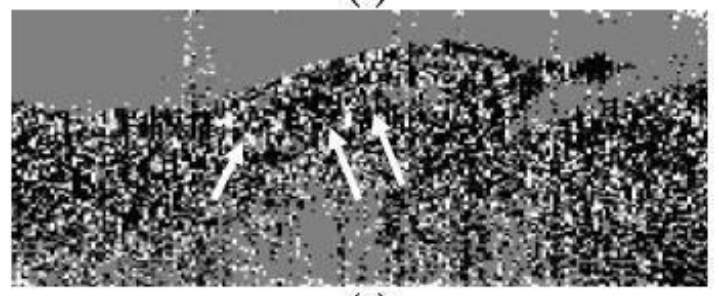

(c)

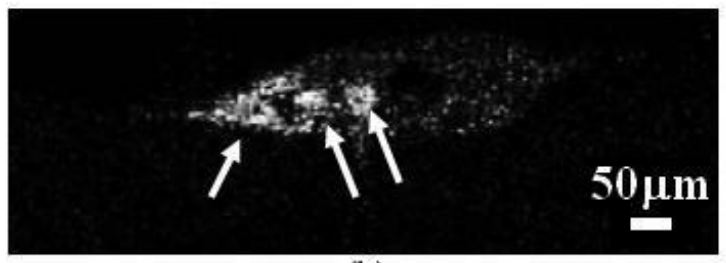

(b)

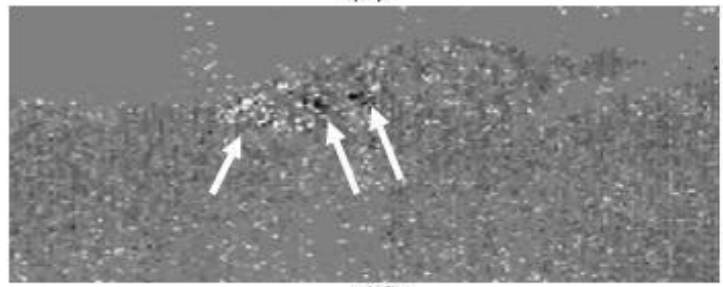

(d)

Figure 7. MB-scan images of $4 \mathrm{dpf}$ zebrafish tail. (a) OCT intensity averaged image. (b) Phase contrast image using $\mathrm{T}_{2}=1 \mathrm{~ms}$ and $\mathrm{T}_{1}=40 \mu$ s. Conventional Doppler OCT technique images average phase change between successive A-scans over (c) 10 and (d) 100 total scans per transverse position. The image scale in (c) and (d) corresponds to $+/-200 \mu \mathrm{m} / \mathrm{s}$. White arrows in all motion contrast images depict the locations of phase contrast identified in (b). Dorsal side of fish is identified on the right-hand side of images. Data shown uses 200 transverse pixels with an image size is $900 \mu \mathrm{m} \times 325 \mu \mathrm{m}$.

The data shown in figure 7 acquired data over 200 transverse locations over $1.6 \mathrm{~s}$ total acquisition time with acquisition over $100 \%$ of the scan cycle. There was no processing or filtering applied to the shown images beyond the previously described data analysis techniques. The locations identified with the white arrows in figure 7 (b) appear to correspond to the features identified in the histological image of figure 6(c) labeled as the dorsal aorta, the axial vein and the gut. These three regions can be at least partially observed in the Doppler image in figure 7 (d), which suggests that this is true motion and not a computational artifact. Since the zebrafish is living in the imaging environment, the motion observed at the locations of the expected blood vessels can be understood. In this case, it appears that the zebrafish is in a stage where motility in the gut region can be observed as well.

The observation of blood vessels is best demonstrated with the comparison between figures 7(b) and 7(d). The Doppler image with increased statistics demonstrates the required SNR-limiting phase noise suppression required to view any flow in this case, where flow is nearly perpendicular to the imaging 
direction. Figure 7(b) demonstrates that through the phase variance, motion is observed over a much larger spatial region than in the Doppler image, resulting in improved visualization.

The BM-scan data shown in figure 8 was taken over the same scan region on the zebrafish tail as the previously demonstrated MB-scan. In this case, with 512 transverse pixels and acquisition over only $50 \%$ of the scan cycle, the phase contrast image uses a phase separation time $\mathrm{T}_{2}=40 \mathrm{~ms}$. Due to the increased time between phase measurements used in this contrast implementation, conventional Doppler images were subject to artifacts due to phase wrapping. The arrows in figure 8(b) highlight contrast areas corresponding to the dorsal aorta, the axial vein and the gut regions identified using the MB-scan data. The blood vessel contrast locations in this case appear to have lensing artifacts to create shadow contrast artifacts below the expected motion.

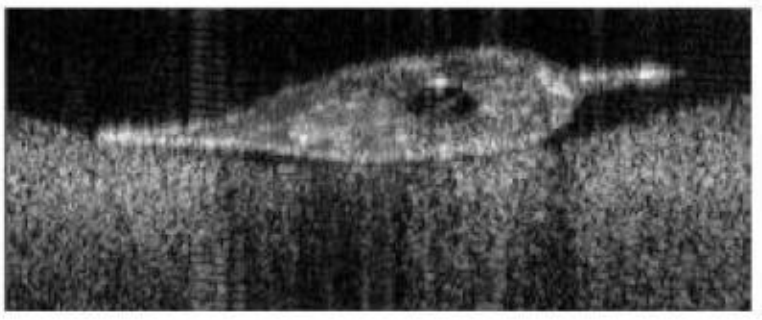

(a)

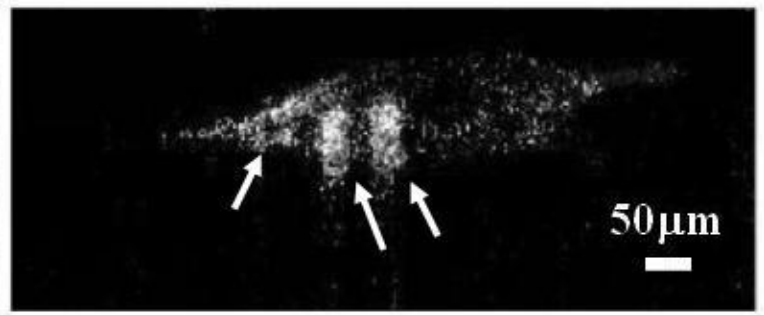

(b)

Figure 8: BM-Scan images of $4 \mathrm{dpf}$ zebrafish tail over same region as used for figure 7. (a) OCT averaged intensity image. (b) Phase contrast image using $\mathrm{T}_{2}=40 \mathrm{~ms}$. Dorsal side of fish is aligned to right hand side of images. Locations of motion contrast indicated with white arrows. Data shown uses 512 transverse pixels with an image size is $815 \mu \mathrm{mx}$ $325 \mu \mathrm{m}$.

In comparing these two phase contrast methods, the MB-scan and the BM-scan have both demonstrated the ability to identify the motion associated with zebrafish vasculature not easily visualized using Doppler OCT techniques. In the ever-increasing push towards faster imaging, speed of these techniques is probably one of the most valid comparisons. While the MB-scan has sufficient statistics of phase information to be able to analyze the phase motion in more ways than just the phase variance, the increased statistics come at the cost of speed. The 200 transverse pixel MB-scan image in figure 7 was acquired in 1.6 seconds, which is much slower than an ideal speed required for in vivo imaging applications. To decrease the amount of imaging time significantly with this method would reduce the motion contrast observed in the image.

There is a lot more flexibility of adjusting parameters of the BM-scan. Reducing the total number of statistics used for phase contrast will reduce the total imaging time without severely reducing contrast. Applying image filtering can be used to compensate for the lack of image smoothing effects due to decreased statistics. With simply reducing the number of phase changes used with BM-scan phase contrast images, the data shown in figure 8 (b) could be acquired in 0.2 seconds. Improving the efficiency of the acquisition during the scan cycle can also bring an improvement to the total acquisition time, but possibly at the cost of some motion contrast due to a reduced time between phase measurements. To keep the same motion contrast with this improved efficiency, a larger pixel scan image can be acquired in the same acquisition time. Improved phase noise analysis can also be used to reduce phase noise in the BM-scan phase contrast image and will be pursued in future improvements of this project.

\section{CONCLUSIONS}

We have demonstrated two different phase contrast approaches capable of imaging zebrafish vasculature in cases where the axial component of flow is very small. The MB-scan and the BM-scan techniques shown have the ability to identify locations of high scatterer motility and can be implemented with a wide range of imaging parameters to adjust imaging size and speed of acquisition. 


\section{REFERENCES}

[1] Fercher AF, Drexler W, Hitzenberger CK, Lasser T, Reports on Progress in Physics 66 (2): 239-303 (2003)

[2] Leitgeb RA, Schmetterer L, Hitzenberger CK, Fercher AF, Berisha F, Wojtkowski M, Bajraszewski T, Optics Letters 29 (2): 171-173 (2004)

[3] Park B, Pierce MC, Cense B, Yun SH, Mujat M, Tearney GJ, Bouma BE, de Boer JF, Optics Express 13 (11): 3931-3944 (2005)

[4] Makita S, Hong Y, Yamanari M, Yatagai T, Yasuno Y, Optics Express 14 (17): 7821-7840 (2006)

[5] Yazdanfar S, Yang CH, Sarunic MV, Izatt JA, Optics Express 13 (2): 410-416 (2005)

[6] Vakoc BJ, Yun SH, de Boer JF, Tearney GJ, Bouma BE, Optics Express 13 (14): 5483-5493 (2005)

[7] The Zebrafish Information Network - www.zfin.org

[8] Isogai, S, Horiguchi, M, Weinstein BM, Developmental Biology, Vol. 230, 278-301 